\title{
Cambiando el pasado: ventajas de la retrocausación*
}

\author{
Hernán Miguel y Rolando Núñez Pradenas**
}

\begin{abstract}
Resumen
Desde sus orígenes, la mecánica cuántica nos ha enfrentado a una serie de "misterios" que se desprenden de ella si es que consideramos esta teoría científica desde una perspectiva realista. En los primeros años del desarrollo de la teoría, científicos de la talla de Albert Einstein notaron las consecuencias de aceptar una teoría como esta, la que permitiría fenómenos como la no-localidad. Esto llevó a una parte de la comunidad científica a considerar que la mecánica cuántica era una teoría incompleta, pues debían existir variables que pudieran explicar los fenómenos "perturbadores" tales como la correlación entre partículas acopladas.
\end{abstract}

Se han propuesto diferentes interpretaciones de la mecánica cuántica que han intentado develar o explicar la realidad subyacente a su formalismo. Una de estas interpretaciones es la llamada "Interpretación Transaccional" de la mecánica cuántica, defendida principalmente por el físico John G. Cramer. Según esta interpretación, los eventos cuánticos se entienden como interacciones causales entre ondas retrasadas viajando hacia adelante en el tiempo y ondas avanzadas viajando hacia atrás en el tiempo. Esta interpretación abre la puerta para aceptar un modelo de causación hacia el pasado o retrocausación.

Autores como Phil Dowe o Huw Price han mostrado una postura favorable a un modelo de retrocausación por la capacidad de resolver efectivamente algunas de las características más perturbadoras derivadas de la mecánica cuántica. A pesar de que el costo intuitivo es bastante alto, la retrocausación es una de las interpretaciones posibles de los resultados de la correlación entre partículas acopladas que nos provee un marco explicativo con algunas ventajas interesantes.

En este trabajo exploramos tales ventajas pues la retrocausación nos otorga un modelo explicativo generalizable para todos los casos de este tipo y presupone procesos y entidades que no quedan solamente en el campo especulativo sino que permitirían ciertas posibilidades de testeo. Rescataremos también la importancia de modelos explicativos no predictivos como ha sido el caso en

\footnotetext{
* Recibido: mayo 2016. Aceptado: junio 2016.

** Universidad de Buenos Aires. Buenos Aires, Argentina. Email: ciencias@retina.ar /

Universidad de Concepción. Concepción, Chile. Email: rolandonunez@udec.cl
} 
otros campos de las ciencias naturales, siempre que no resulten ad hoc gracias a las restricciones que impiden su aplicación a cualquier caso.

Palabras clave: Retrocausación, mecánica cuántica, no-localidad, explicación.

\begin{abstract}
Since its inception, quantum mechanics has faced a series of "mysteries" that emerge from it if we consider this scientific theory from a realistic point of view. In the early development of the theory, scientists like Albert Einstein noticed the consequences of accepting a theory like this, which allow phenomena such as non-locality. This led a part of the scientific community to believe that quantum mechanics was an incomplete theory, since there should be variables that might explain those "disturbing" phenomena such as the correlation between entangled particles.

Different interpretations of quantum mechanics have been proposed that have tried to reveal or explain the reality underlying its formalism. One of those interpretations is the "Transactional Interpretation" of quantum mechanics, advocated mainly by physicist John G. Cramer. According to this interpretation, quantum events are understood as causal interactions between delayed waves traveling forward in time and advanced waves traveling backward in time. This interpretation opens the door to accept a model of causation to the past or retrocausation.
\end{abstract}

Authors like Phil Dowe or Huw Price have shown a favorable attitude toward the model of retrocausation because it seems to be capable of effectively solve some of the most disturbing features derived from quantum mechanics. Despite the intuitive cost is rather high, retrocausation is one of the possible interpretations for the results of the correlation between entangled particles to provide us with an explanatory framework with some interesting advantages.

In this paper we explore such advantages, as the retrocausation gives us a generalizable explanatory model for all cases of this type and assumes processes and entities that are not only in the field of speculation but allow certain possibilities of testing. Also, we will rescue the importance of notpredictive explanatory models as has been the case in other fields of natural sciences, provided that those model do not be ad hoc due to restrictions that prevent its application to any case.

Keywords: Retrocausation, quantum mechanics, non-locality, explanation. 


\section{Introducción}

Hemos visto cómo es que desde sus orígenes, la mecánica cuántica nos ha enfrentado a una serie de "misterios" que se desprenden de ella si es que consideramos esta teoría científica desde una perspectiva realista. En los primeros años del desarrollo de la teoría, científicos de la talla de Albert Einstein notaron las consecuencias de aceptar una teoría como ésta, la que permitiría fenómenos como la no-localidad. Esto llevó a una parte de la comunidad científica a considerar que la mecánica cuántica era una teoría incompleta, pues debían existir variables que pudieran explicar los fenómenos "perturbadores" tales como la correlación entre partículas acopladas.

Ya en sus inicios, se han propuesto diferentes interpretaciones de la mecánica cuántica que han intentado develar o explicar la realidad subyacente a su formalismo, y nos concentramos en una de estas interpretaciones, la llamada "Interpretación Transaccional" de la mecánica cuántica, defendida principalmente por el físico John G. Cramer (1986). A partir de esta interpretación, los eventos cuánticos podrían ser entendidos como interacciones causales entre ondas retrasadas viajando hacia adelante en el tiempo y ondas avanzadas viajando hacia atrás en el tiempo. Notamos cómo esta interpretación abre la puerta para aceptar un modelo de causación hacia el pasado o retrocausación.

Autores como Phil Dowe (2000) o Huw Price (1997) han mostrado una postura favorable a un modelo de retrocausación por la capacidad de resolver efectivamente algunas de las características más perturbadoras derivadas de la mecánica cuántica. A pesar de que el costo intuitivo es bastante alto, la retrocausación es una de las interpretaciones posibles de los resultados de la correlación entre partículas acopladas que nos provee un marco explicativo con algunas ventajas interesantes. Por este motivo, nos hemos concentrado particularmente en el modelo de la retrocausación, y en trabajos previos de uno de nosotros, ${ }^{1}$ vimos cómo es que sería posible llegar a concebirlo desde la perspectiva de la cognición corporeizada. Sin embargo, pudimos notar también que dada la enorme relevancia que tiene la experiencia en la formación de conceptos en la cognición corporeizada, se hace necesario contar con alguna manera de acceder experiencialmente a algo así como un fenómeno retrocausal, que bajo el modelo de la interpretación transaccional, eso no es posible. Adicionalmente, en mecánica cuántica no vamos a poder realizar mediciones sin, por ejemplo, hacer colapsar el estado de una partícula en un estado medible, de modo que no habría manera de medir sin modificar aquello que se quiere medir.

En este trabajo ahondaremos en la retrocausación pues ésta podría otorgarnos un modelo explicativo generalizable para algunos de los casos más complejos de la mecánica cuántica, y plantearemos que la retrocausación podría presuponer procesos y entidades que no quedan solamente en el campo especulativo sino que permitirían arreglos experimentales en los que podrían ser medidos. Será esta última posibilidad la que nos permitiría aceptar que realmente estamos teniendo alguna experiencia

\footnotetext{
${ }^{1}$ Núñez Pradenas $(2012 ; 2014)$.
} 
empírica comprobable de retrocausación en el mundo microfísico y si podemos efectivamente tener acceso a dicha experiencia, podríamos entonces validar la retrocausación más allá de una mera extensión metafórica del concepto de causa.

\section{Experiencias en el mundo cuántico}

Conocidos son los problemas denunciados por Albert Einstein, Boris Podolsky y Nathan Rosen (1935) a través de la conocida paradoja EPR, en la que se muestra cómo es que la entonces naciente mecánica cuántica presentaba problemas si es que aceptábamos que ésta nos da una representación de la realidad física. Más específicamente, Einstein y compañía concluyen su ya famoso trabajo de 1935, según el cual la mecánica cuántica seguramente debía ser, en su estado de desarrollo a la fecha, una teoría incompleta, pues si aceptamos que bajo su formalismo subyace cierta realidad, tenemos que aceptar ciertos fenómenos perturbadores o poco intuitivos, como la existencia de partículas acopladas. Esto no sólo es problemático explicativamente, sino que además entraría en contradicción con la física relativista. Por esto es que Einstein pensó que debía existir algún elemento faltante en esta descripción de la realidad que pudiera dar cuenta de todas las consecuencias involucradas en la mecánica cuántica sin tener que aceptar, por ejemplo, algún mecanismo de acción a distancia. Einstein y compañía, por lo tanto, apelaban a que la cuántica no podía ser entendida todavía como una teoría completa. Sin embargo, en 1964, el trabajo realizado por John Bell echó por tierra las pretensiones de Einstein y sus coautores, pues demostró, a través del teorema que lleva su nombre, que ninguna teoría de variables ocultas podía producir las predicciones de la mecánica cuántica, a menos que dejara de lado el realismo o la localidad.

Los resultados de Bell han sido corroborados por pruebas experimentales en varias ocasiones, siendo uno de los casos más conocidos el de los experimentos de Alan Aspect (et al.) en 1981. En este punto estamos hablando de experiencias con respecto al mundo microfísico y no solo de cuestiones de formalismo. De hecho, podemos notar el cambio de estatus, el real salto ocurrido desde la formulación del teorema de Bell a la confirmación empírica a partir de las experiencias del tipo Aspect. Si bien aceptamos el formalismo del teorema, la corroboración de la experiencia es la que genera el convencimiento en las personas. Debido a lo relevante de este punto para nuestro trabajo es que analizaremos con un poco más de detalle este tipo de experiencias.

En caso de querer emular los resultados de Aspect para poder entender las experiencias a nivel del universo microfísico en cuántica, debiésemos proceder de la siguiente manera. Supongamos que contamos con un generador de pares de fotones que arroja como resultado una tasa constante de $40 \%$ de fotones con polarización horizontal ${ }^{2}$. Ahora, sometiendo estos fotones al experimento de Aspect, polarizamos uno del par, y obtenemos una alta correlación con que el otro fotón del par muestre esa misma polarización. Estos son los extraños resultados de la mecánica cuántica a

\footnotetext{
${ }^{2}$ Esta no es exactamente la configuración usada por Aspect, pero inspirados en su histórico experimento, imaginamos este otro para poder echar luz sobre la retrocausación.
} 
los que Einstein se refiere en la paradoja EPR que señalamos más arriba, en los que la acción sobre uno de los fotones del par, está altamente correlacionada con el valor de la misma característica del otro fotón del par.

A continuación, una vez configurada la fuente, registramos una línea más o menos constante a la altura de un $40 \%$ en el gráfico del porcentaje de fotones con la característica de polarización horizontal. Supongamos adicionalmente que se muestra un ruido, o existe una dispersión, digamos, del orden del 1\%.

Ahora bien, llevamos ahora adelante el experimento tipo Aspect con el generador de partículas configurado de esa manera y utilizando pares de fotones de modo que algunos pares tomarán parte en un grupo (A) y otros en un grupo diferente (B). Un punto crucial es que la pertenencia de los pares a cada uno de los grupo, A o B, se produce de modo aleatorio. Para algunos pares de fotones, a uno de los fotones del par se lo somete al estímulo que lo hace tomar un valor en particular de polarización. Esta acción sucede sin que todavía se asigne el par al grupo A o al grupo B. Por lo cual esta intervención en uno de los fotones del par todavía no permite distinguir si se trata de un fotón del grupo A o del grupo B. Una vez realizada esta intervención, se produce la asignación al azar de manera que los pares de fotones queden en el grupo A o en el grupo B.

Si el par de fotones ha sido asignado al grupo A o al grupo B, tendrá un tratamiento diferente. Si el par fue asignado al grupo A y uno de sus fotones ya ha sido intervenido como indicamos más arriba, se medirá el valor de polarización del otro fotón de ese par.

Mientras que, si el par de fotones ha sido asignado al grupo B, no se realizará ninguna intervención en el otro fotón del par. En resumen, a uno de los fotones del par se lo somete al estímulo que lo hace tomar un valor en particular de polarización, mientras que al otro fotón del par, luego del proceso aleatorio de asignación al grupo A o al grupo B, se le aplica o no la medición de su polarización, respectivamente. Como consecuencia, a los pares que han sido asignados al grupo A, se les ha medido la polarización a ambos fotones del par. Mientras que a aquellos pares que resultaron asignados al grupo B, solo a uno de los fotones del par se le ha medido su polarización.

Como ya anticipamos, hay una muy alta correlación entre el resultado de la medida de polarización entre uno y otro de los dos componentes del par. Esto indica que lo que ya fue señalado sigue en discusión, y es que no hay un marco teórico adecuado a la intuición para dar cuenta de por qué lo que ocurre a un componente del par está correlacionado con lo que ocurre al otro componente del par. Esta es una de las raíces de las diversas interpretaciones a las que da lugar la mecánica cuántica.

Podíamos vernos inclinados a pensar que los resultados se explican por alguna variable oculta previa, por ejemplo, una causa común en el pasado. Sin embargo, no podremos atribuir esto a una causa común ya que en el principio los fotones no tienen determinado todavía su valor de polarización y, además, la correlación no es del 100\%. Pero el detalle más importante aparecería al nivel de las predicciones estadísticas. En los pares del grupo (A), aquellos en los que hubo interacción con ambos fotones del par, encontramos el esperado $40 \%$ de polarización horizontal. Sin embar- 
go, en el grupo (B), en donde esperaríamos tener esa misma proporción, encontramos que hay solo un $30 \%$ de polarización horizontal en el componente del par que ha sido medido, habiendo dejado evolucionar al otro componente del par sin medirlo.

El resultado no esperado desde la visión clásica es que haya una merma en el porcentaje del grupo (B), habiendo sido generados estos pares con el generador en las mismas condiciones que para el grupo (A) y habiendo sido separados por un proceso al azar.

\section{Análogo macroscópico: El caso de los gemelos}

Para clarificar las cosas, si es que eso se aplica en este tipo de experimentos y resultados, explicaremos esta experiencia con un análogo macroscópico. Este ejemplo análogo es presentado por Phil Dowe (2000: 180) para presentar los problemas de la paradoja EPR. Modificaremos ligeramente el ejemplo de Dowe para adaptarlo a nuestra investigación. Supongamos la existencia de un par de hermanos gemelos que viven en una misma ciudad de clima no muy frío. Uno de ellos decide mudarse a Ushuaia, una ciudad de clima muy frío de Argentina, en donde cae enfermo afectado por una patología desencadenada por las bajas temperaturas y muere. Un grupo de investigadores comienzan a notar cierta correlación entre el clima frío y la muerte de ciertos individuos, debido a que se observa muy a menudo que, cuando un par de gemelos van a una ciudad de clima frío, ambos mueren, al punto que se podría hablar de una correlación uno-a-uno, en donde al someter a dos gemelos a un clima frío, uno muere si y sólo si el otro también muere. Dentro de la comunidad científica comienza a desarrollarse la hipótesis de que existiría una condición genética causante de la enfermedad, la que puede ser rastreada al momento anterior a que los gemelos se separan en el vientre de su madre, por lo que si uno tiene esta condición, el otro también la tendrá. Esta sería en este caso la hipótesis de la causa común planteada para el ejemplo de los fotones antes visto.

Teniendo en cuenta estos datos, se plantea el siguiente experimento. Se convocan a parejas de gemelos (idénticos genéticamente) a participar del experimento. Una vez iniciado el procedimiento, un par de gemelos es llevado al aeropuerto y cada uno de los gemelos es subido a aviones diferentes. Uno de los aviones va siempre en dirección a Punta Arenas, una cuidad de clima muy frío en Chile. El otro avión va en dirección a Ushuaia, una ciudad de Argentina también de clima muy frío, pero cuando este avión despega, el piloto del avión debe lanzar una moneda, y según el resultado sea cara o cruz, sigue su rumbo a Ushuaia o vuelve al Aeropuerto ubicado en Santiago (fig. 1). En cuanto los aviones llegan a su destino, cualquiera de ellos sea, los individuos son encerrados y monitoreados. Los científicos simplemente toman nota acerca de si los participantes mueren o no. 


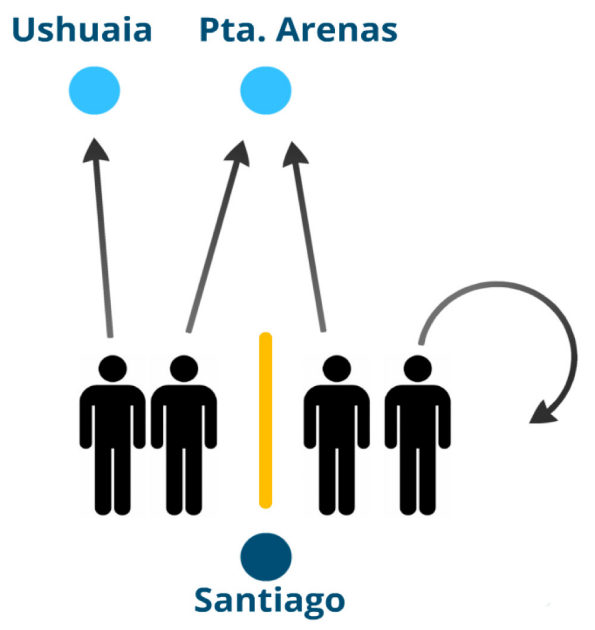

Fig. 1.

Tenemos entonces dos grupos, los pares Ushuaia - Punta Arenas y los pares Punta Arenas - Aeropuerto (Santiago). En el caso del primer grupo, los investigadores encuentran que el $60 \%$ de los gemelos que fueron llevados a Ushuaia murieron y el $60 \%$ de los gemelos que fueron llevados a Punta Arenas murieron. El 40\% restante en ambos casos sobrevive sin mayores problemas. Este resultado muestra una correlación uno-a-uno entre quienes murieron en Ushuaia y quienes murieron en Punta Arenas, lo que para la comunidad de investigadores confirmaría la teoría del mecanismo genético como causa de esta enfermedad (y por lo tanto, como causa común de la muerte de los gemelos). Pero en el caso del grupo Punta Arenas - Santiago los resultados difieren de manera interesante. El 45\% de los individuos que fueron llevados a Punta Arenas murieron, mientras que ninguno de los que regresaron al aeropuerto murió (fig.2). Este último resultado es esperable pues estamos hablando de individuos que no fueron sometidos a condiciones de frío, pero el descenso en la tasa de mortalidad en los individuos de este grupo llevados a Punta Arenas en comparación con los individuos del grupo anterior es enigmático, porque si la teoría genética es correcta, el hecho de que un gemelo sea o no llevado a una ciudad fría no debería afectar en nada los resultados de la prueba en el otro gemelo. Notaremos que estamos frente a otra versión del experimento de Aspect, en donde parece ser que la realización del test (específicamente, el que sea llevado o no a un lugar frío) en uno de los gemelos va a influir de manera no-local en el resultado del test del otro gemelo (el que sí fue llevado a Punta Arenas mientras su gemelo volvía al Aeropuerto). 


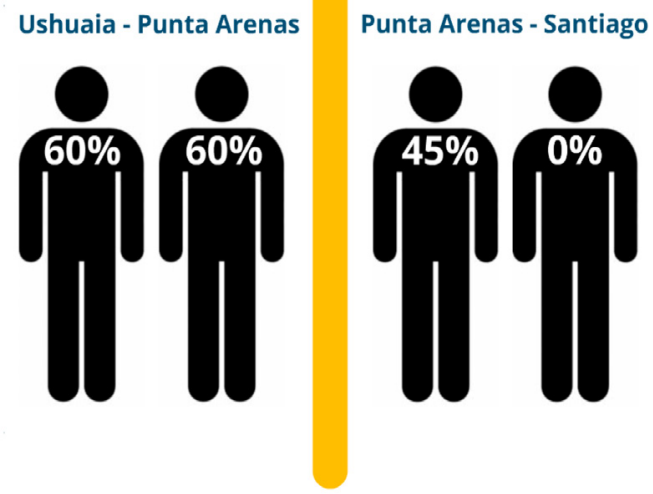

Fig. 2.

En pocas palabras, nos queda la imagen de que, lo que le ocurre a un componente del par influye en lo que le ocurra al otro componente del par y esa influencia no puede ser explicada con intuiciones sencillas. Para este resultado se han esgrimido muchas perspectivas, correspondientes con las distintas interpretaciones. Planteamos ya desde el comienzo que una de estas perspectivas es la retrocausación, y ésta no ha sido considerada con suficiente detenimiento quizás por la tradición reinante en la física respecto del rechazo a una relación de causación que no coincida con el principio de causalidad y la preferencia a que los procesos se ajusten a una flecha temporal acorde a la evolución de los sistemas en los que tienen lugar los distintos procesos.

La retrocausación puede dar cuenta de estos resultados, tanto para el ejemplo de los fotones como para el análogo macroscópico de los gemelos, de la siguiente manera. El componente del par que no ha sido sometido a estímulo, en el presente $t_{1}$ tiene ciertas características de modo que influye modificando el origen del par en el instante $t_{0}$, en la fuente (en el pasado). Este paso es claramente un paso de causación hacia atrás en el tiempo. A partir de esa modificación es esperable que el otro componente del par, aquél que en $\mathrm{t}_{1}$ ha sido efectivamente medido, muestre esta modificación.

Con este argumento queda explicado por qué el grupo B muestra una proporción menor que el grupo A. En nuestra versión macroscópica, esto equivaldría a sostener que, el que uno de los gemelos no sea sometido a la prueba del frío (los aviones que regresan a Santiago) causará en parte la condición genética en el vientre materno, lo que luego influirá en la baja del porcentaje de muertes en las mediciones sobre el otro gemelo.

Hasta aquí, la propuesta retrocausal que permite explicar no solo esta merma sino que además la no coincidencia para los casos en que no hubo correlación, más difíciles de encuadrar desde, por ejemplo, una visión holista. Por otro lado, el modelo retrocausal se muestra altamente compatible con la idea de causalidad física propuesto por Dowe basado en el traspaso de cantidades conservadas. Por supuesto, en el caso del ejemplo de los fotones, tenemos no-localidad sólo si consideramos lo que ocurre 
con ambos fotones en $t_{1}$, pero no hay no-localidad si tomamos en consideración el traspaso desde $\mathrm{t}_{1} \mathrm{a}_{0} \mathrm{y}$ luego de vuelta $\mathrm{a} \mathrm{t}_{1}$, porque todo se explica por el traspaso de las ondas retrasadas y avanzadas, lo que en principio sería compatible con la física relativista (Dowe 2000:185). Aún cuando esta solución no está exenta de problemas, esta propuesta no enfrenta las dificultades de compatibilizar la cuántica con la relatividad.

\section{Testeando la retrocausación}

Queremos ahora someter a discusión la siguiente propuesta en esta misma línea. ¿Si la propuesta de retrocausación fuera correcta, qué otros datos la apoyarían? Porque no podemos decir que su único apoyo es aquél resultado anómalo que intentamos iluminar. Si así fuera, no dejaría de ser un recurso ad hoc, que solo ha sido pergeñado para reconciliar los resultados con el marco conceptual. Debe recordarse que la filosofía de la ciencia tiene en muy baja estima este tipo de recursos, aun cuando la historia de la ciencia y la práctica científica actual muestra casos en los que el éxito se ha debido en parte a la defensa recalcitrante de ciertas hipótesis ad hoc, incluso antes de que fueran confirmadas por evidencia adicional a los resultados que le dieron origen.

Obviamente la propuesta retrocausal se vería apoyada si se hubiera obtenido en $\mathrm{t}_{0}$ un porcentaje menor. Se tendría que haber registrado en $\mathrm{t}_{0}$ la característica en cuestión para decidir si aparecen o no rastros del primer tramo causal, esto es de $t_{1}$ a $t_{0}$. Pero, en caso de que en $\mathrm{t}_{0}$ ya estuviera presente el valor de polarización en cuestión, diríamos que se trata de un caso de causa común, ya sea que se midan uno o ambos fotones del par. Este problema no es tal, más bien es una diferencia entre el resultado para el par en cuestión y los resultados estadísticos. Si contáramos con que en $\mathrm{t}_{0}$ ha mermado la proporción estadística, digamos al $35 \%$, por ejemplo, no tenemos motivos para esperar que se obtengan resultados diferentes en los grupos A y B, digamos de $40 \%$ en el grupo A y $30 \%$ en el grupo B. Ya que la asignación a tales grupos es aleatoria. Si la configuración inicial muestra un $35 \%$ de la característica en cuestión, no tiene ningún sentido que al distribuir los pares en dos grupos al azar, uno de los grupos muestre el $40 \%$ y el otro muestre el $30 \%$ de esa característica. En ese caso se pondría en duda la aleatoriedad del mecanismo. En cambio lo contrario es correcto: si sabemos que hubo $40 \%$ en el grupo A y $30 \%$ en el B, esperamos que el lote total muestre un $35 \%$ de ese rasgo.

En términos del ejemplo de los gemelos, si el hecho de que sometiéramos o no a uno de los gemelos a la prueba de frío es causa de la condición genética generada en el vientre materno antes de la separación de los gemelos, la que luego afectaría a su hermano, los investigadores deberían ser capaces de encontrar esa condición genética al momento del nacimiento de los gemelos (o en el útero materno). Si esto fuese así, los investigadores considerarían que la causa de la enfermedad que afecta a ambos gemelos es esta condición, no el hecho de que uno de ellos haya sido sometido a una prueba en el futuro (fig. 3a). Sin embargo, si esto fuese así, y considerando las cifras antes expuestas por los pares, los investigadores deberían haber registrado la condición genética en un 52,5\% de las madres en el pasado. Pero esto no explicaría por qué tenemos dos grupos, uno de $60 \%-60 \%$ y otro de $0 \%-45 \%$, porque lo que 
esperaríamos es que ambos grupos estuviesen equilibrados, pues la asignación de grupos se hace por medio del azar (fig. 3b). Con esto dejamos totalmente de lado la tesis de la causa común previa.

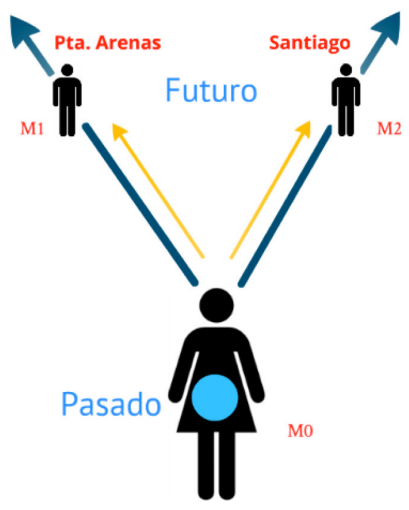

Fig. 3a.

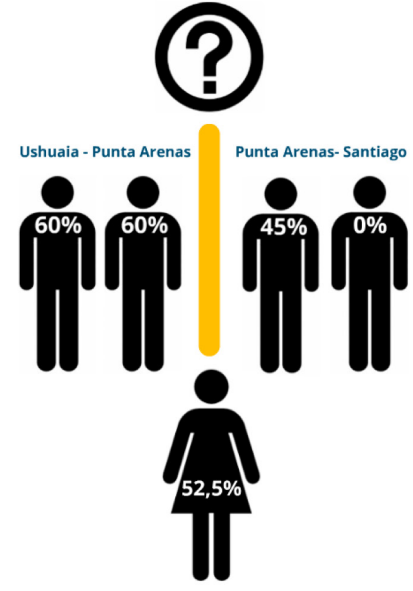

Fig. 3b.

Dejando el análogo macroscópico, el problema por el cual los resultados estadísticos en $\mathrm{t}_{0}$ no explican los resultados dispares entre los dos lotes en $\mathrm{t}_{1}$ es que la asignación de un par a uno u otro de los grupos es un proceso azaroso, de modo que este proceso no puede distinguir entre las características preexistentes y asignar pares con mayor chance de cierto resultado al grupo A y con menor chance de ese mismo resultado al grupo B. Dicho esto, queda claro que si tuviéramos el registro estadístico de las chances de cierto resultado en $\mathrm{t}_{0}$ podríamos obtener una confirmación del proceso de retrocausación.

\section{Midiendo sin intervenir}

El problema hasta ahora insalvable es que al medir en $\mathrm{t}_{0}$, el valor obtenido de la medida queda determinado y de ese modo toda otra medida posterior no puede dar resultados diferentes. Se trata del problema del colapso de la función de onda o bien de la decoherencia en el proceso de medición. Aquí debemos abandonar, al menos en parte, nuestro análogo macroscópico, pues este problema no estaría presente en el caso de los gemelos, ya que ahí podríamos intentar hacer una medición que quedara registrada sin generar una intervención mayor ${ }^{3}$. De todos modos, más adelante retomaremos el ejemplo por cuestiones explicativas.

Ahora bien, para poder continuar con nuestra propuesta, consideraremos la posibilidad de estimar las chances mediante una interacción no decoherente. Esto es

\footnotetext{
${ }^{3}$ De todos modos vale la pena destacar que podríamos adaptar el análogo macroscópico si, por ejemplo, estableciéramos que la medición misma les produciría a los gemelos la baja resistencia al frío o algo por el estilo. Sin embargo, para efectos de la exposición del problema, no parece necesario tener que continuar desarrollando este ejemplo.
} 
algo que hasta el momento no ha sido posible de desarrollar, pero que en física se ha estado trabajando para poder obtener resultados que vayan en esta línea. Por ejemplo, uno de los métodos que se ha utilizado para poder realizar algo como esto es poder 'reconstruir' la información posteriormente. Por supuesto, la salvedad de esto es que no tenemos la muestra original. Inmediatamente podemos notar que reconstruir no es lo mismo que poder medir sin hacer colapsar, y esto va a ser un problema no menor, pero por ahora vamos a continuar considerando que esto pueda llegar a realizarse.

Tomemos en consideración el ya clásico experimento de la doble rendija. El propósito original del experimento mental de la doble rendija en su formulación moderna es mostrar la dualidad onda-corpúsculo tanto de la luz como de la materia. El experimento se plantea de la siguiente manera. Supongamos que enviamos un haz de partículas en dirección a una pantalla, y entre la fuente y la pantalla ponemos una doble rendija. En una de las rendijas pondremos un detector, de manera tal que si el detector está encendido y por lo tanto es capaz de interactuar con las partículas, éstas pasarán por alguna de las dos rendijas comportándose como partículas al hacer impacto en la pantalla (fig. 4a). Sin embargo, si el detector está inactivo, entonces cada partícula pasará en forma de onda por el arreglo de ambas partículas y aparecerá un patrón de interferencia en la pantalla (fig. 4b).

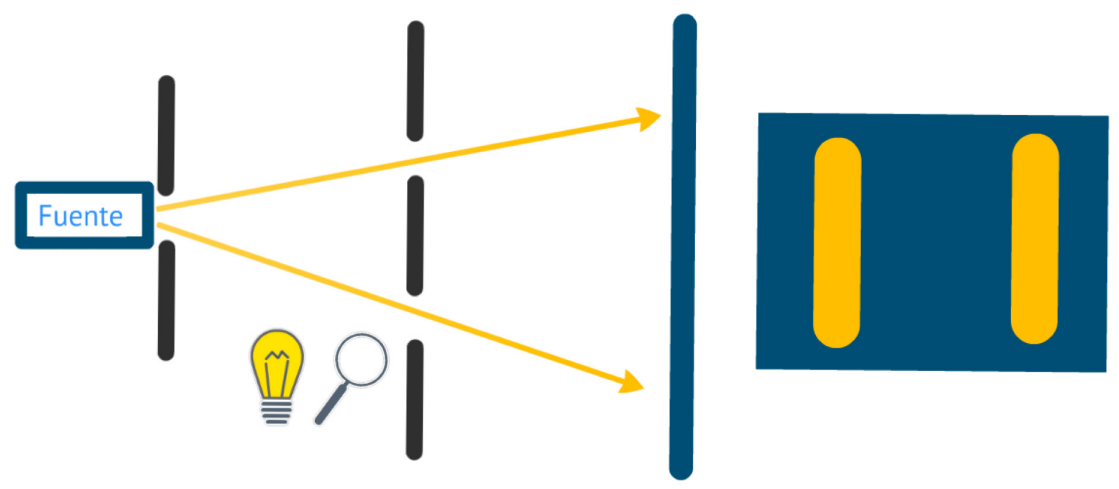

Fig. 4a.
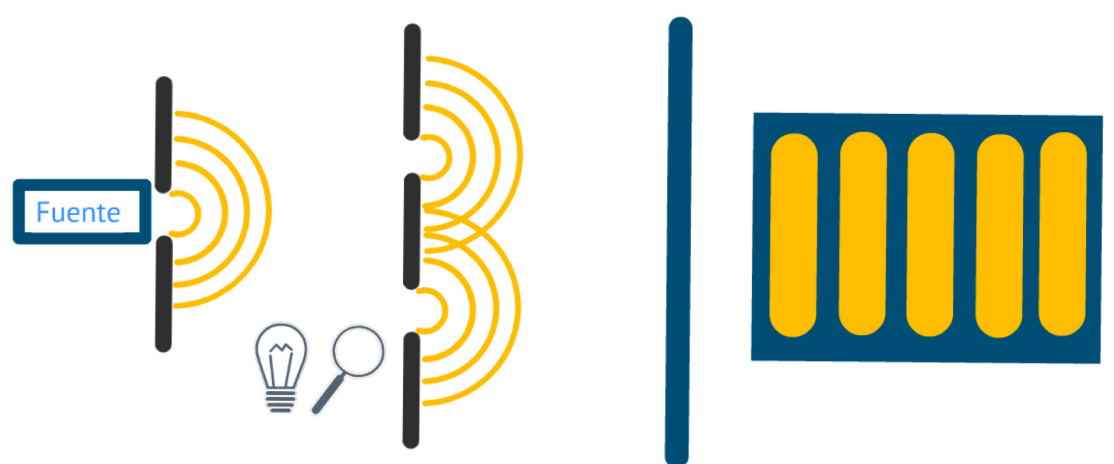

Fig. 4b. 
Vale la pena destacar que el experimento mental ha sido respaldado por experiencias en laboratorios, en principio sólo con luz, pero ya desde 1961 con electrones. Este experimento fue realizado por el físico alemán Claus Jönsson (1974) en la Universidad de Tübingen. Incluso ya desde 1974, el equipo de físicos italianos conformado por P. G. Merli, G. F. Missiroli y G. Pozzi (1976) lograron realizar el experimento ya no con chorros de electrones, sino que con electrones individuales, pudiendo demostrar que cada electrón interfiere consigo mismo, resultado ya predicho en la cuántica.

Ahora bien, sumémosle a este experimento que el detector es cuántico, de modo que si efectivamente interactúa produciéndose su decoherencia por el paso de la partícula, queda inhabilitado para nuevas interacciones. Este tipo de detector es al que nos referíamos al mencionar la posibilidad de que exista una interacción no decoherente. Ahora precisemos que el detector está ubicado para interactuar de modo decoherente con las partículas que pasen por la rendija de la derecha, pero su interacción con las que pasan por la izquierda no produce su decoherencia.

A partir de este nuevo ensamble tenemos tres resultados posibles: a) una partícula al pasar muestra un patrón de interferencia como si no hubiera detector (fig. 5a), mostrando que el detector se encuentra inactivo; b) una partícula pasa por la rendija inferior y hace colapsar el estado del detector (el detector se ha disparado, fig. 5b), mostrando un patrón simple en la pantalla; y finalmente c) una partícula pasa por la rendija superior y el estado del detector no tuvo decoherencia (quedó sin dispararse, fig. 5c). Nos interesa la situación c) porque hemos obtenido la medición de que el detector está activo y sin embargo no ha sido disparado, con lo cual está en condiciones de una próxima interacción en la que se dispararía.

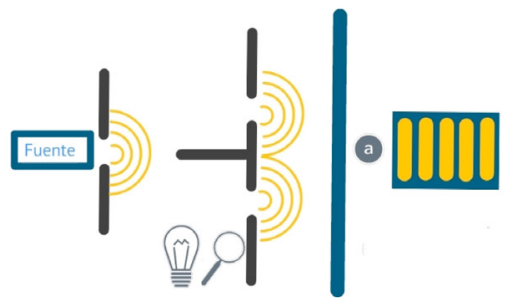

Fig. 5a.

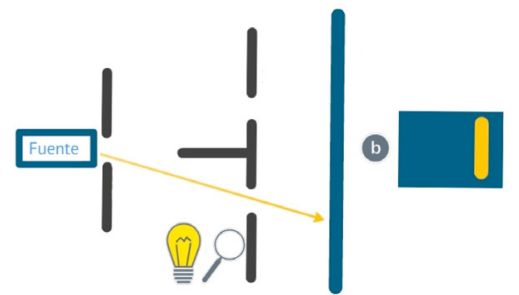

Fig. 5b.

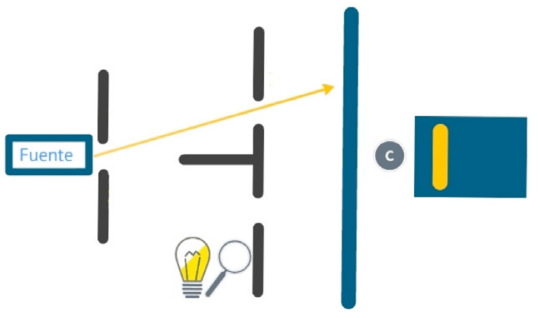

Fig. 5c. 
A continuación, tomemos este recurso de medición cuántica del caso c) en la que no se ha producido el colapso de la función de onda o interacción no decoherente y traslademos esta situación a nuestro experimento anterior. Tomemos una fuente de pares de fotones que los genera con direcciones de polarización en diferente dirección y proporción. Armemos ahora un arreglo como en los experimentos de Aspect.

Sometamos a cada par de fotones a la medición cuántica no decoherente de modo que filtramos los fotones y nos quedamos solamente con los que han pasado el test en las condiciones c), suponiendo que ese test detecta fotones que pueden dar por resultado polarización horizontal. Mantenemos el generador de partículas con esta configuración y utilizamos para el experimento tipo Aspect de los grupos A y B, los fotones obtenidos como resultado de la interacción no decoherente mencionada en c). Lo que estamos haciendo es tomar los fotones de los cuales ya tenemos la medida, hemos elaborado una medición previa que nos permite tener registro de los fotones sin que se haya producido colapso. Supongamos entonces que contamos con un haz de pares de fotones cuya chance de resultar polarizados horizontalmetne es del $40 \%$. A partir de ahora realizamos el experimento y analizamos todo retrospectivamente, para facilitar la descripción en pasado y evitar aparentes contrasentidos lingüísticos como "esto ocurrió hoy debido a lo que ocurrirá mañana" lo cual dificulta las intuiciones.

Una de las predicciones de la propuesta retrocausal es que en $\mathrm{t}_{0}$, en el pasado, tiene que haber habido una caída al 35\% en algún lapso correspondiente al proceso en el que tuvo lugar el experimento con los grupos A y B que resultaron mostrar $40 \%$ y $30 \%$ respectivamente y con cantidades similares de mediciones. Esto se explica por lo mismo que le exigíamos al análogo macroscópico, de modo que si en el registro del pasado no apareciera tal merma, no habría rastros en $t_{0}$ de lo que tuvo lugar en $\mathrm{t}_{1}$ (de lo que tuvo lugar en el futuro) y eso contaría como una anomalía que debería ser tenida en cuenta por parte de los defensores de la propuesta retrocausal. Si en cambio registramos esa merma, tal depresión en el gráfico no tiene causa aparente en modificaciones de la configuración del equipo y por tanto quedaría como un comportamiento anómalo. Para peor, como ya se dijo anteriormente, esta merma es compatible con el lote completo de A y B, pero no puede ser la causa de que el grupo A muestre el resultado al 40\% y el grupo B al 30\% ya que cada par es asignado aleatoriamente y no por alguna interacción entre el proceso aleatorio y su chance para cierto resultado. Si tal disminución apareciera en el gráfico de origen, entonces no contaríamos con cuáles fueron las causas previas que la generaron ni ella misma podría ser causa de los resultados posteriores de los lotes A y B. En cambio, su existencia quedaría perfectamente enmarcada como efecto de la distribución diferente de A y B, y no esperaríamos que el porcentaje de origen diera lugar a A y B. Es decir, no se produce ningún loop causal sino solamente un tramo de retrocausación que sirve para dar cuenta de la existencia de la disminución del porcentaje de origen y así dar cuenta también del resultado en el par que ha sido medido. Tal característica echaría luz sobre el problema estadístico y sobre el problema de que un componente del par pueda influir en la chance del resultado a obtener en el otro componente.

Podemos volver a nuestro análogo macroscópico para poder explicar en términos más sencillos el caso de los fotones, pero hay que dejar establecido de antemano que 
este tipo de correlaciones no se dan en el mundo macroscópico, y que por lo tanto no hay soporte empírico en esa escala que pueda avalar este tipo de experiencias. Por supuesto, tampoco es nuestra intención avalar aplicaciones de resultados de la mecánica cuántica de este tipo de casos. El uso que haremos del análogo macroscópico es sólo con fines explicativos.

Presentadas las advertencias pertinentes, recordemos que en nuestro análogo teníamos dos grupos de pares de gemelos, los pares Punta Arenas-Ushuaia y los pares Punta Arenas-Santiago (aeropuerto). Lo que vamos a agregar al caso es la posibilidad de realizar una medición en la fuente, que en el caso de los fotones correspondía a $t_{0}$. En este caso, estaríamos hablando de un punto en el que los gemelos compartían el útero materno, digamos en 1981, año en que fueron gestados los participantes del estudio que se realiza en 2012 (fig. 6). Recordemos que para el caso de los fotones tuvimos que apelar a la existencia de interacción no decoherente, mientras que aquí eso no será necesario ${ }^{4}$.

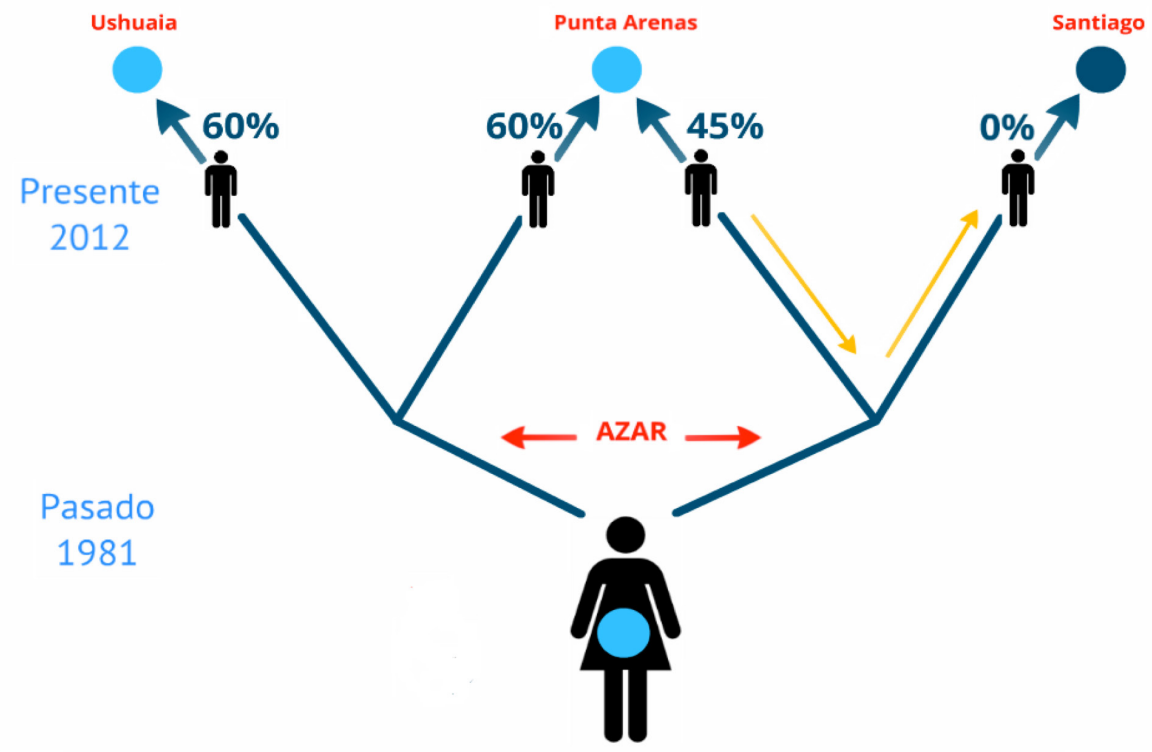

Fig. 6.

Gracias a la posibilidad de mediciones realizadas en el pasado, pensemos que en años previos al nacimiento de los gemelos se comenzó a llevar registro a través de estudios intrauterinos de si los niños venían con algún problema genético que los hiciera especialmente vulnerables al frío. Los registros marcaron una sostenida tendencia a través de los años que indicaba que un $60 \%$ de los gemelos traían esa condición. Los gemelos participantes del experimento del viaje a Punta Arenas en 2012 fueron sometidos a las pruebas intrauterinas (nuestro equivalente a $\mathrm{t}_{0}$ para el caso de los fotones) en el año de su gestación, digamos, 1981. En ese año los investigadores

\footnotetext{
${ }^{4}$ Esto marca también los límites de esta analogía dado que no nos encontramos con las dificultades reales de la mecánica cuántica, pero las advertencias ya han sido establecidas.
} 
notaron una baja en la tasa de gemelos que traían la condición de hipersensibilidad al frío que llegó al 52,5\%. En los años posteriores, los registros volvieron a su habitual $60 \%$. Lo interesante de esto es que esa disminución en la tasa queda explicada por los experimentos que se realizarán en el futuro a los gemelos y no viceversa, y un modelo retrocausal puede hacerse cargo de la distinción entre los grupos Ushuaia-Punta Arenas y Punta Arenas-Santiago, ya que si fuese un caso de causa común, los resultados del experimento realizado en 2012 debiesen haber sido de un parejo 52,5\% ya que la división de los grupos fue aleatoria, mientras que si postulamos que efectivamente el que uno de los gemelos no haya sido sometido al clima frío causó algo en la fuente, podemos explicar la antes misteriosa e inexplicada disminución en 1981 de la tasa de gemelos con hipersensibilidad al frío.

Nótese que no hace falta proponer ningún tramo de retrocausación para dar cuenta de los resultados en el caso de los fotones del lote A, y tampoco estos procesos parecen tener lugar en un nivel macroscópico (más allá de nuestro caso análogo, que tiene una función explicativa para el desarrollo del trabajo), de manera que la presencia de tramos de retrocausación no es en sí mismo un artilugio ad hoc que pueda aplicarse a cualquier episodio vacuamente explicativo.

\section{Conclusiones}

La noción de retrocausación puede aplicarse para dar cuenta de algunos de los procesos peculiares de la mecánica cuántica de manera exitosa.

No es necesario proponer tramos de retrocausación en todos los procesos para dar cuenta de los resultados, por lo cual la inclusión de tramos de retrocausación no parece ser un recurso ad hoc. Su uso parece adecuado para los casos en que el resto de las propuestas se muestra deficiente.

$\mathrm{Su}$ inclusión en las explicaciones causales no viola ninguno de los principios vigentes en nuestras mejores teorías y permite inferir procesos que, por otra parte, no son indetectables en principio.

Este análisis cuenta con que se mantengan expresamente separadas las asimetrías causales de las temporales, aun cuando puedan coincidir en la mayoría de los casos.

Adicionalmente, este análisis propone un modo en el que la discusión científica y filosófica sobre la causación, la temporalidad y su interacción pueda abordarse de modo enriquecedor.

Queda pendiente una seria discusión acerca de qué ocurre si intentamos conocer el registro obtenido en $\mathrm{t}_{0}$ echándole una mirada en un tiempo intermedio entre $\mathrm{t}_{0} \mathrm{y}$ $\mathrm{t}_{1}$. Tal discusión no puede abordarse sin una topología temporal adecuada no lineal con ramas de evolución de los sistemas en la dirección temporal y otras en dirección contraria, pero este análisis excede el marco del presente trabajo y no afecta los resultados de lo presentado hasta aquí.

Por otro lado, si vamos a aceptar tramos de retrocausación a este nivel, debiésemos estar preparados para hacer frente a algunos de los problemas clásicos de la causación hacia el pasado, y es la posibilidad de que una vez hecha la primera medi- 
ción (la realizada en $\mathrm{t}_{0}$ o la realizada en útero), pareciera que los investigadores involucrados en los experimentos del futuro no serían libres de elegir no realizar dichos experimentos, no sin dejar los resultados de la primera medición no explicados. Este es el problema de las cadenas causales cerradas, que quedará para otra oportunidad.

\section{Referencias bibliográficas}

Aspect, Alain; Grangier, Philippe; Roger, Gérard (1981): "Experimental Tests of Realistic Local Theories via Bell's Theorem" en Physical Review Letters. 47 (7): 460-463.

Bell, John (1964): "On the Einstein Podolsky Rosen Paradox" en Physics, 1 (3): 195-200.

CRAmer, John 1(986): "The Transactional Interpretation of Quantum Mechanics" en Reviews of Modern Physics, 58: 647-687.

Cramer, John (1998): "An Overview of the Transactional Interpretation of Quantum Mechanics" en International Journal of Theoretical Physics, 27: 227-236.

Dowe, Phil (1997): "A Defense of Backward-in-Time Causation Models in Quantum Mechanics" en Synthese, 112: 233-246.

Dowe, Phil (2000): Physical Causation. New York: Cambridge University Press.

Einstein, Albert; Podolsky, Boris; Rosen, Nathan (1935): "Can Quantum-Mechanical Description of Physical Reality Be Considered Complete?" en Physical Review, 47 (10): 777-780.

NúÑEz Pradenas, Rolando (2012): "Consideraciones epistemológicas sobre la retrocausación" en Velasco, M. y Venturelli, N. (Eds.) Epistemología e Historia de la ciencia. Selección de trabajos de las XXII Jornadas de Epistemología e Historia de la Ciencia, 18: 412-429.

NúÑEz Pradenas, Rolando (2014): "Cognición corporeizada, tipos de causación y mecánica cuántica" en Hernán Miguel (Eds.) Explicación, Causación y Contrafácticos. Buenos Aires, Editorial Prometeo: 61-78.

Price, Huw (1996): Time's Arrow and Archimedes's Point. New York: Oxford University Press.

Penrose, Roger (2006): El camino a la realidad. Barcelona: Debate.

Reichenbach, Hans (1999): The Direction of Time. New York: Dover Publications.

ZeH, Heinz-Dieter (2001): The Physical Basis of The Direction of Time. Berlin: Springer-Verlag. 